\title{
Mix de medios como estrategia clave para la promoción de productos lácteos
}

Media mix as a key strategy for the promotion of dairy products

\author{
Villavicencio Rodas María Fernanda \\ Universidad Técnica de Machala \\ mvillavicencio@utmachala.edu.ec \\ Machala - Ecuador \\ Jaramillo Gonzalez Mayra Lucia \\ Universidad Técnica de Machala \\ mljaramillo_est@utmachala.edu.ec \\ Machala - Ecuador
Gallegos Calderón Evelyn Katiuska
Universidad Técnica de Machala
egallegos2@utmachala.edu.ec
Machala - Ecuador

\author{
Luis Joel Sánchez Saritama* \\ Universidad Técnica de Machala \\ 1sanchez5@utmachala.edu.ec \\ Machala - Ecuador
}

\section{Resumen}

Analizar los hábitos de consumo de amas de casa del cantón Pasaje, en relación a los medios digitales y tradicionales que determinen la efectividad de una campaña promocional para productos lácteos. Muestreo probabilístico estratificado, amas de casa de 15 a 44 años. El 48,0 \% de encuestadas utilizan ambos medios, el 30,5\%; medios digitales; el $21 \%$ medios tradicionales y el $0,5 \%$ no utilizan medios. Un eficiente mix de medios para promocionar productos lácteos, necesita aplicar estrategias en medios digitales y tradicionales a nivel nacional y local.

Palabras clave: Mix de medios, medios digitales-tradicionales, amas de casa. 


\section{Abstract}

To analyze the consumption habits of housewives of the Pasaje canton, in relation to digital and traditional media that determine the effectiveness of a promotional campaign for dairy products. Stratified probability sampling, housewives aged 15 to 44 years. Results: $48.0 \%$ of respondents use both means, $30.5 \%$; digital media; $21 \%$ traditional media and $0.5 \%$ do not use media. An efficient media mix to promote dairy products needs to apply strategies in digital and traditional media at the national and local level.

Keywords: Media mix, digital-traditional media, housewives

\section{Introducción}

Las organizaciones direccionan todos sus esfuerzos de marketing para captar la atención de su público objetivo, pero no consideran la importancia de seleccionar los medios de comunicación que se adapten a su segmento que pueden ser online y offline. (Terrones, 2018). Mediante el presente escrito las empresas podrán establecer estrategias promocionales en múltiples medios según sus objetivos, podrán dirigirse a un segmento importante en la actualidad como lo es el género femenino. El cantón Pasaje de la Provincia de "El Oro" y según (Instituto Nacional de Estadísticas y Censos, 2020 ), el cantón Pasaje registra el $49.47 \%$ de habitantes mujeres, misma que resultan un total de 36014 Ciudadanas. Por lo tanto, surge la pregunta que motiva esta investigación: ¿El comportamiento del consumidor en cuanto al consumo de medios tradicionales y digitales condiciona la efectividad de la estrategia promocional para productos lácteos y sus derivados dirigida a las amas de casa del cantón Pasaje?. El objetivo principal de la presente investigación, es analizar los hábitos de consumo de las amas de casa del cantón Pasaje, en relación a los medios digitales y tradicionales que determinen la efectividad de una campaña promocional para productos lácteos y con los resultados se pretende dar respuesta a las siguientes hipótesis. H1: La efectividad de la estrategia promocional para productos lácteos y sus derivados dirigida a las amas de casa del cantón Pasaje, está condicionada por el comportamiento de consumo de medios y H2: El mix de medios es una estrategia clave para la promoción de productos lácteos y sus derivados dirigida a las amas de casa en el Cantón Pasaje.

\section{Mix de medios}

El mix de medios es indispensable en la comunicación de una empresa para presentar su producto a los clientes, por ello el autor (Ortiz, 2016) lo plantea como una base de comunicación integral en la cual la empresa puede llegar de una manera mucho más rápida y eficaz a su público definido, esto se puede dar mediante el desarrollo y los clientes, es decir se encarga de identificar los atributos más importantes del producto o servicio que brinde la empresa, para poder mostrárselos al cliente de tal manera que este se sienta persuadido por este tipo de información. Se conoce que más del $70 \%$ de la personas se encuentran migradas a los medios digitales mientras que el restante aún 
permanecen fijos a medios tradicionales, por eso este autor cree conveniente realizar una campaña de mix de medios con publicidad en medios digitales donde se encuentra gran parte del mercado, pero también tomar en cuenta los medios tradicionales para no olvidar ese nicho que también podrían ser clientes para la empresa. (Minodora \& Raluca, 2017)

\section{Medios tradicionales en el Ecuador}

Según (Cardador Cabello, 2019) los medios tradicionales son los medios de comunicación más utilizados antes de la globalización del internet, es decir todos aquellos medios como: televisión, radio, revistas, etc., Estos fueron usados para todas las actividades informativas y muchas veces de comercialización como fueron la televisión o la radio. Dentro del contexto local los medios tradicionales en el Ecuador formaron parte de nuestra sociedad mucho tiempo más que en el resto del mundo, al ser un país tercermundista y con graves problemas económicos y políticos. En los tiempos actuales y con la mayor facilidad que la tecnología ha permitido expandirse a cada rincón del mundo. (Gehrke, Lizarazo, Noboa, Olmos, \& Pieper, 2016)

\section{Medios digitales en el Ecuador}

El entorno empresarial y los diversos medios de comunicación, han apostado por invertir y desenvolverse en las redes sociales y todas las funciones relacionadas a ello (Vivar Flores, 2019). Según la información del INEC, se analizó la conectividad existente en el Ecuador, donde se abarca el acceso a internet y comunicación móvil. El INEC demuestra que el 36,0\%de los hogares cuenta con conexión a internet. Un estudio de medios realizado por Mentinno, una consultora de marketing ecuatoriana; detalla que en Ecuador 13,8 Millones cuentan con acceso a internet; además en cuanto a redes sociales, existen 12,04 millones de perfiles en Facebook, 4,02 millones de usuarios en Instagram, continúa Tik Tok con 1,4 millones de usuarios, 0,9 millones para twitter 880.000 usuarios, mientras que Snapchat tiene 285000 usuarios y Spotify cuenta con 3'610.000; Messenger cuenta con 7’700.000 y finalmente 8’100.000 para WhatsApp. (Juan Del Alcázar , 2020)

\section{Comportamiento del consumidor}

Para el éxito de una campaña promocional es necesario conocer cómo se comportan los consumidores en los diferentes medios de comunicación (online y offline) es por esto que es primordial definir qué factores influyen en el cliente al momento de efectuar una compra. El comportamiento del consumidor son las acciones por parte de los consumidores al momento de adquirir productos o servicios, la toma de decisiones, sus gustos y sus exigencias, que son predominadas por la motivación y la experiencia de los compradores, siendo estos los impulsores a la acción de comprar, que anteceden y establecen el modelo de compras que tiene una persona (Mercado, Perez, Castro, \& Macias, 2019).

También se refiere al conjunto de acciones orientadas a satisfacer las necesidades, deseos y requerimientos de las personas a través de productos y servicios. Las necesidades que son solventadas en su mayoría tienen que ver con los aspectos básicos y fisiológicos que 
intervienen en la vida cotidiana del hombre, es así que el comportamiento del consumidor es un estudio muy amplio que abarca los hábitos de consumo, frecuencia y volumen de compra (Gallegos, 2016).

\section{Materiales y métodos}

Para realizar la identificación de la muestra se ha considerado los datos del total de población de mujeres para el cantón Pasaje comprendidas en el rango de edad de 15 a 44 años de edad, publicados por (Alcaldía del Cantón Pasaje, 2015), y partiendo desde esos datos se realizó la proyección de la población femenina el año 2019, considerando un tasa de crecimiento de población del 1,5\% publicado por el (INEC, 2010).

\section{Calculo de la muestra}

\begin{tabular}{|c|c|}
\hline Parámetro & Valor \\
\hline $\mathrm{N}$ & 37634 \\
\hline $\mathrm{Z} 2$ & 3,8416 \\
\hline $\mathrm{P}$ & 0,5 \\
\hline $\mathrm{Q}$ & 0,5 \\
\hline e2 & 0,00252004 \\
\hline
\end{tabular}

El resultado después del cálculo para la proyección es que existe una población de mujeres de 37634 mujeres en el cantón Pasaje para el año 2019; este dato a su vez es considerado para el cálculo de la muestra con un $95 \%$ de confianza y un margen de error del 5\%; que se realiza mediante la siguiente fórmula:

$$
\begin{gathered}
n=\frac{N * Z_{\alpha}^{2} * p * q}{e^{2} *(N-1)+Z_{\alpha}^{2} * p * q} \\
n=\frac{37634 * 3,8416 * 0,5 * 0,5}{0,00252004 *(37634-1)+3,8416 * 0,5 * 0,5} \\
n=\frac{36143,6936}{95,79706532} \quad n=\approx 377
\end{gathered}
$$

Finalmente la muestra comprende a 377 mujeres dentro de las edades de 15 años a 44 años de edad, considerando que las mujeres en la edad de 15 años en adelante comienzan a incluir en sus rutinas diarias actividades domésticas y por ende empiezan a tomar decisiones de compra pequeñas en sus hogares. Con muestreo probabilístico estratificado, por afijación simple asignando un número de encuestas de manera equitativa para todas las parroquias con preguntas cerradas y abiertas. 


\section{Resultados}

Los medios de comunicación tanto digitales como tradicionales tienen una gran incidencia en el comportamiento de las amas de casa del cantón Pasaje, lo que refleja que el 30,5\% interactúen en medios digitales de una manera eficaz como por ejemplo en redes sociales y el $21 \%$ aún se mantiene en medios tradicionales. Por tanto, se deben ejecutar estrategias promocionales en función a los medios digitales para lograr la comercialización de productos lácteos y sus derivados.

Dentro de los medios tradicionales podemos concluir que en el medio de TV, el canal más seleccionado es Ecuavisa con el 45,4\% cuyo contenido que observan las amas de casa es informativo con el $35,8 \%$, es medio es visto "casi siempre" con el 35,8\% por lo regular en la jornada nocturna con el 44,2\%. En Radio, la más sintonizada es La Otra con el 32.3\%, prefieren el contenido informativo con el $33.1 \%$, pero la escuchan "a veces" con el $31,5 \%$ en la mañana con el 53,1\%. La emisora con menor sintonía por parte de las amas de casa es Radio Machala con el $0.8 \%$.

El 89,2\% resalto que no leen ninguna revista en particular, por otra parte Diario El Correo es el periódico con mayor demanda por parte de las amas de casa con un $24,7 \%$, siendo el contenido informativo con el 63,8\% la razón de su consumo. Dentro de los medios digitales, las redes sociales son tendencia como medio de comunicación actual por ello la red social más utilizada es Facebook con un 55,3\%, su frecuencia de uso es siempre con un 47,3\%. Seguido de la plataforma digital más utilizada esta YouTube con un 69,2\% de uso entre las demás plataformas.

Al reconocer como tendencias las redes sociales podemos rescatar que las personas utilizan este tipo de medios para el propio interés personal con 19,80\%, es decir como entretenimiento mucha de las veces, un $53.69 \%$ de las personas suelen ver lo que publican otras personas o páginas y en ocasiones interactúan por medio de comentarios, esto permite que el $38.3 \%$ de los encuestados estén de acuerdo que las redes sociales influyen de manera importante en la vida diaria, pero son evaluadas como buenas en un 45,6\%, ya que permiten comunicarse de manera fácil y sencilla según el $62.5 \%$ de las personas investigadas. En función de los resultados explicados anteriormente, se determina el perfil del consumo mix de medios en las amas de casa del cantón Pasaje:

Tabla 1.

Perfil de la mujer frente a los medios tradicionales

\begin{tabular}{|l|l|l|l|l|l|}
\hline \multirow{5}{*}{ Televisión } & Edad & Canal & Frecuencia & Jornada & Contenido \\
\cline { 2 - 6 } & $15-19$ & $\begin{array}{l}\text { Pasaje Tv } \\
\text { Ok tv }\end{array}$ & Casi siempre & Todo momento & $\begin{array}{l}\text { Informativo } \\
\text { Series }\end{array}$ \\
\cline { 2 - 6 } & $20-24$ & $\begin{array}{l}\text { TC } \\
\text { Ecuavisa }\end{array}$ & Siempre & Mañana & $\begin{array}{l}\text { Informativo } \\
\text { Entretenimiento }\end{array}$ \\
\cline { 2 - 6 } & $30-34$ & Ecuavisa & Casi siempre & Mañana & $\begin{array}{l}\text { Informativo } \\
\text { Entretenimiento }\end{array}$ \\
\cline { 2 - 6 } & $35-39$ & Ecuavisa & Siempre & Noche & Informativo \\
\hline
\end{tabular}


ISSN: 2588-056X

\begin{tabular}{|c|c|c|c|c|c|}
\hline & & & & & Telenovelas \\
\hline & $40-44$ & Ecuavisa & Casi siempre & Noche & $\begin{array}{l}\text { Informativo } \\
\text { Películas }\end{array}$ \\
\hline \multirow{6}{*}{ Radio } & Edad & Emisora & Frecuencia & Jornada & Contenido \\
\hline & $15-19$ & Ninguna & A veces & Tarde & Entretenimiento \\
\hline & $20-24$ & La otra & Casi siempre & Mañana & Entretenimiento \\
\hline & $30-34$ & La otra & Casi siempre & Mañana & Entretenimiento \\
\hline & $35-39$ & Ninguna & Siempre & Mañana & Entretenimiento \\
\hline & $40-44$ & $\begin{array}{l}\text { Superior Fm } \\
\text { La otra } \\
\text { Ninguna }\end{array}$ & $\begin{array}{l}\text { Siempre } \\
\text { Casi siempre } \\
\text { Nunca }\end{array}$ & Mañana & Informativo \\
\hline \multirow{6}{*}{ Periódico } & Edad & Diario & Contenido & & \\
\hline & $15-19$ & Opinión & Informativo & & \\
\hline & $20-24$ & El correo & Informativo & & \\
\hline & $30-34$ & $\begin{array}{l}\text { Correo } \\
\text { El nacional }\end{array}$ & Informativo & & \\
\hline & $35-39$ & El nacional & Informativo & & \\
\hline & $40-44$ & El nacional & Informativo & & \\
\hline
\end{tabular}

Elaboración: los autores

Tabla 2.

Perfil de la mujer frente a los medios digitales

\begin{tabular}{|l|l|l|l|l|l|}
\hline & Edad & $\begin{array}{l}\text { Plataforma } \\
\text { Digital }\end{array}$ & Red Social & Comportamiento & Frecuencia \\
\cline { 2 - 6 } & $15-19$ & $\begin{array}{l}\text { Netflix } \\
\text { YouTube }\end{array}$ & $\begin{array}{l}\text { WhatsApp } \\
\text { Facebook }\end{array}$ & $\begin{array}{l}\text { Observan, comentan } \\
\text { y comparten }\end{array}$ & Siempre \\
\cline { 2 - 6 } $\begin{array}{l}\text { Medios } \\
\text { digitales }\end{array}$ & $20-24$ & $\begin{array}{l}\text { YouTube } \\
\text { Netflix }\end{array}$ & $\begin{array}{l}\text { Facebook } \\
\text { WhatsApp }\end{array}$ & Observan & Siempre \\
\cline { 2 - 6 } & $30-34$ & $\begin{array}{l}\text { YouTube } \\
\text { Netflix }\end{array}$ & $\begin{array}{l}\text { Facebook } \\
\text { Instagram }\end{array}$ & $\begin{array}{l}\text { Observan, comentan } \\
\text { y comparten }\end{array}$ & Siempre \\
\cline { 2 - 6 } & $35-39$ & $\begin{array}{l}\text { YouTube } \\
\text { Netflix }\end{array}$ & $\begin{array}{l}\text { Facebook } \\
\text { WhatsApp }\end{array}$ & Observan & $\begin{array}{l}\text { Casi } \\
\text { siempre }\end{array}$ \\
\cline { 2 - 6 } & $40-44$ & $\begin{array}{l}\text { YouTube } \\
\text { Facebook Watch }\end{array}$ & $\begin{array}{l}\text { Facebook } \\
\text { WhatsApp }\end{array}$ & Observan & Siempre \\
\hline
\end{tabular}

Elaboración: los autores 


\section{Conclusiones}

El comportamiento del consumidor condiciona la efectividad de las estrategias promocionales, puesto que los medios de comunicación tanto digitales como tradicionales tienen una gran incidencia en el comportamiento de las amas de casa del cantón Pasaje; por lo tanto, es de suma importancia ejecutar estrategias promocionales adaptadas a las plataformas más influyentes de ambos medios de comunicación, para lograr obtener excelentes resultados en la comercialización de productos lácteos y sus derivados.

La H1 es válida, debido a que se identificó que la implementación estratégica de un mix de medios, crea efectividad en las estrategias promocionales que la empresa quiera impartir a su segmento meta. Las mujeres se encuentran atraídas por medios de comunicación digital, debido a que están de acuerdo en que los medios influyen de alguna manera en su vida diaria, siendo persuadidas por información que ven o escuchan en aquellos medios por otra parte, hay amas de casa que casi siempre observan la Tv en horario de la noche, y a veces por las mañanas sintonizan la radio. Lo que permite deducir que incluir ambos medios en una estrategia de comunicación para lácteos tendrá excelentes resultados si se emplea adecuadamente el tiempo y se utilizan herramientas de diseño y creatividad. Se sugiere elaborar una campaña publicitaria acerca de los lácteos y sus derivados, a través de medios tradicionales ( $\mathrm{T} v$ y radio), y medios digitales (redes sociales) que es donde se encuentra el público objetivo al que se quieren llegar.

La H2 es válida, ya que se determinó que en la actualidad la mujer es la protagonista principal en la decisión de compra de productos del hogar, lácteos y sus derivados. Las empresas que realizan la venta de los productos antes mencionados y que desean comercializarlo en el cantón Pasaje y captar un nuevo segmento como lo son las amas de casa, deben implementar un mix de medios estratégico que incluya medios tradicionales como digitales el cual direccionen las estrategias promocionales del producto al segmento que se desea llegar.

\section{Referencias}

Alcaldía del Cantón Pasaje. (15 de Febrero de 2015). Plan de Desarrollo y Ordenamiento Territorial. Obtenido de http://app.sni.gob.ec/snilink/sni/PORTAL_SNI/data_sigad_plus/sigadplusdocumentofinal/07600007700 01_PDOT\%20PASAJE\%20ACTUALIZADO\%202015_15-03-2015_23-1011.pdf

Cardador Cabello, A. L. (2019). Gestión del marketing 2.0. Antequera: IC Editorial.

Gallegos, D. (2016). El valor percibido por el cliente y el comportamiento del consumidor como constructos paralelos a las Leyes de Gossen. Revista Oikos: Revista de la Escuela de Administración y Economía. Obtenido de https://dialnet.unirioja.es/servlet/articulo?codigo $=5841090$ 
Gehrke, M., Lizarazo, N., Noboa, P., Olmos, D., \& Pieper, O. (2016). Panorama de los medios en Ecuador. Media Development STUDIES.

Granda, C. P., \& Velasquez, A. (2016). La comunicación estratégica digital en las organizaciones públicas de Ecuador. Estado actual y proyección. Revista Latina de Comunicación Social.

INEC. (2010). Instituto Nacional de Estadísticas y Censos. Obtenido de Resultados del censo de población y vivienda El Oro 2010: https://www.ecuadorencifras.gob.ec/wp-content/descargas/Manulateral/Resultados-provinciales/el_oro.pdf

Instituto Nacional de Estadísticas y Censos. (2020 ). Proyección de la poblacion ecuatoriana, por años calendario, según cantones 2010-2020. Quito. Obtenido de https://www.obraspublicas.gob.ec/wpcontent/uploads/downloads/2017/03/proyeccion_cantonal_total_2010202012016-v1.pdf

Juan Del Alcázar . (Enero de 2020). Mentinno: Innovation \& Lifetime Value Partners. Obtenido de Ecuador Estado Digital Ene/20.

Mercado, K., Perez, C., Castro, L., \& Macias, A. (2019). Estudio Cualitativo sobre el Comportamiento del Consumidor en las Compras en Línea. Información Tecnológica, 30(1), 109-120. doi:http://dx.doi.org/10.4067/S071807642019000100109

Minodora, S., \& Raluca, M. (2017). El impacto emocional de los medios tradicionales y los nuevos medios en acontecimientos sociales. Comunicar, 11. Obtenido de https://www.redalyc.org/pdf/158/15849613010.pdf

Ortiz, M. (2016). Gerencia de Marketing. En M. Ortiz, Gerencia de Marketing (pág. 328). Colombia: Universidad del Norte. Obtenido de https://books.google.es/books?hl=es\&lr=\&id=CNJCDwAAQBAJ\&oi=fnd\&pg= PA153\&dq=medios+de+comunicacion+marketing\&ots=2t9ZzZ6tq3\&sig=d4vIa TmmKYlwE4M0hs9JaIehnKk\#v=onepage \&q\&f=false

Sánchez, I., Benitez Luzuriaga, K., \& Benitez Luzuriaga, F. (2017). Mapa de Comunicación de la Provincia de El Oro. Redes, 417-428.

Terrones, A. (2018). Pensamiento dominante, educación y medios de comunicación. Sophia, Colección de Filosofía de la Educación.

Vivar Flores, J. (2019). Nuevos modelos de comunicación, perfiles y tendencias en las redes sociales. 